\title{
Influence of Thermal Annealing on the Electrical Properties of Poly(3-hexylthiophene)-Based Thin Film Diodes
}

\author{
Zivayi Chiguvare ${ }^{\mathrm{a}}$, Jürgen Parisi ${ }^{\mathrm{b}}$, and Vladimir Dyakonov ${ }^{\mathrm{c}}$ \\ ${ }^{a}$ Renewable Energy Programme, Faculty of Engineering, University of Zimbabwe, \\ P. O. Box MP 167, Mt Pleasant, Harare, Zimbabwe \\ b Department of Energy and Semiconductor Research, Institute of Physics, University of Oldenburg, \\ D-26111 Oldenburg, Germany \\ ${ }^{c}$ Faculty of Physics and Astronomy, Bayerische Julius-Maximilians Universität Würzburg, \\ Am Hubland, D-97074 Würzburg, Germany \\ Reprint requests to Prof. J. P.; E-mail: parisi@ehf.uni-oldenburg.de
}

Z. Naturforsch. 62a, 609-619 (2007); received May 23, 2007

\begin{abstract}
The effects of thermal annealing on the bulk transport properties of poly(3-hexylthiophene) (P3HT) were studied by analyzing room temperature current-voltage characteristics of polymer thin films sandwiched between indium tin oxide/poly[ethylene dioxythiophene]:poly[styrene sulfonate] (ITO/PEDOT:PSS) and aluminum electrodes, before and after a thermal annealing step. It was observed that annealing takes place in two steps: (1) Dedoping of the polymer of impurities such as oxygen, remnant solvent, water, leading to a decrease in the conductivity of the film, and (2) thermally induced motion of the polymer chains leading to closer packing, thus, stronger inter-chain interaction and, consequently, increase in conductivity. It was also observed that the ITO/PEDOT:PSS/P3HT hole injection barrier increases on annealing the ITO/PEDOT:PSS/P3HT/Al thin film devices. The implications of impurity dedoping and closer packing on the output characteristics of bulk heterojunction polymer-fullerene thin film solar cells are discussed.
\end{abstract}

Key words: Space Charge Limited Currents; Thermal Annealing; Polymer Semiconductors. PACS numbers: 72.80.Le, 73.40.Sx, 81.40.Gh, 82.35.Cd, 85.30.De.

\section{Introduction}

Conjugated polymers are interesting materials for the fabrication of electrically conducting devices such as light emitting diodes [1], solar cells [2,3], and thin film field effect transistors [4] on flexible substrates. Poly(3-hexylthiophene) (P3HT) has emerged as one of the highly promising materials and is currently subject of intense research. For instance, power conversion efficiencies of solar cells based on P3HTfullerene blends of up to $3.5 \%$ have been reported [5], while P3HT field effect transistors reaching mobilities of 0.05 to $0.1 \mathrm{~cm}^{2} / \mathrm{Vs}$ and on/off current ratios of $>10^{6}$ have been achieved [6]. However, the physics of devices based on P3HT remains only scarcely understood.

It has been observed that the external quantum efficiency of solar cells, employing $\mathrm{P} 3 \mathrm{HT}$ as electron donor and the fullerene derivative [6,6]-phenyl- $\mathrm{C}_{61}$ butyric acid methyl ester (PCBM) as acceptor, improves on annealing the solar cells. The temperature and duration of annealing are particularly important, as they hold for the actual conditions and the procedure of annealing. Padinger et al. [5] obtained the highest increase in power conversion efficiency after annealing their P3HT:PCBM solar cells at $75{ }^{\circ} \mathrm{C}$ for 5 to 6 minutes. The efficiency increased from 0.4 to $2.5 \%$, i. e., over six times the value for as-cast devices. They have attributed the improvement of the short circuit current density, $J_{\mathrm{sc}}$, from 2.5 to $7.5 \mathrm{~mA} / \mathrm{cm}^{2}$ to increased charge carrier mobility in the blend, presumed to be due to enhanced crystallization of the polymer during the annealing process. Enhancement in open circuit voltage and fill factor was assigned to the burning of shunts. Parker [7] reported that heat treatment enlarges the quantum efficiency of the polymer film because of a change in morphology at the polymer electrode interface. The latter has also been considered responsible for the observed rise in performance of polymerfullerene devices [8]. Both PCBM [9] and regioregular P3HT [10] are known to crystallize in thin films under respective appropriate conditions. The significant improvement of performance, stability, and lifetime of P3HT-based devices after an annealing step 


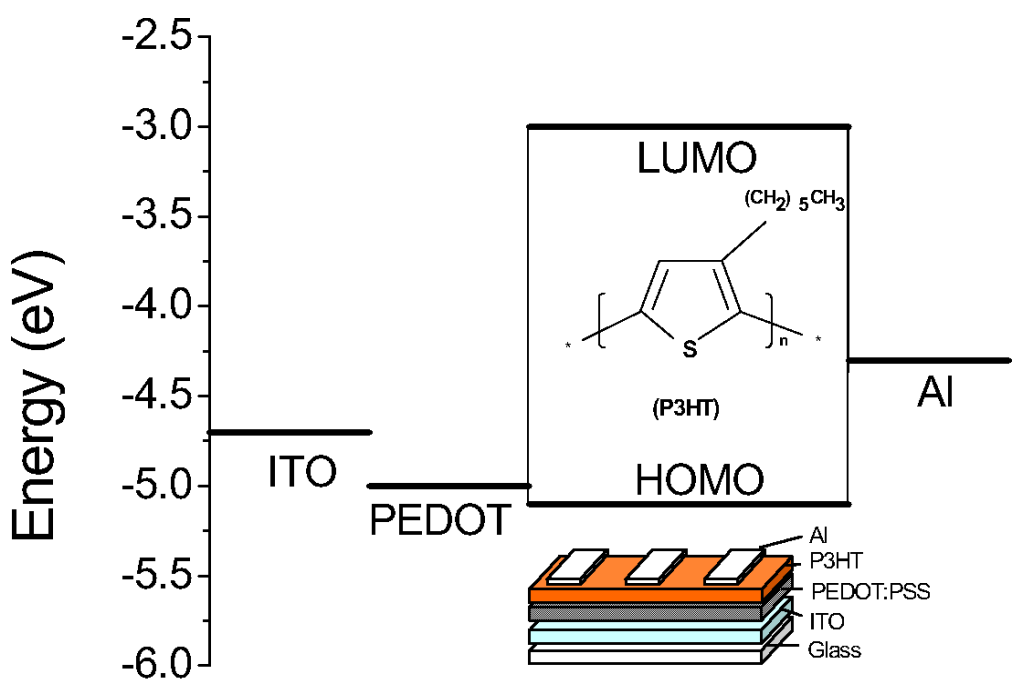

Fig. 1. Energy level diagram of the ITO/PEDOT/P3HT/Al devices studied (under nonequilibrium conditions). The insets show the formula of $\mathrm{P} 3 \mathrm{HT}$ and a schematic diagram of the device structure. performed at temperatures between 60 and $120{ }^{\circ} \mathrm{C}$ has been attributed to the following: crystallization of either PCBM, P3HT, or both; improvement of contact quality; interdiffusion increasing donor-acceptor orbital overlap; film drying by evaporation of moisture and remnant solvent; oxygen dedoping of the polymer; phase separation of donor and acceptor materials; densification/reordering of the polymer film, etc. $[5,11-13]$.

However, it remains still unclear whether the proposed reordering of P3HT would really lead to crystallization and, hence, to an improvement in hole mobility, if one considers the different annealing conditions reported by various authors. So far, it is an open question, why the output characteristics improve upon annealing the device, whether or not crystallization of $\mathrm{P} 3 \mathrm{HT}$ is good for the solar cell, what role is played by PCBM and by the indium tin oxide (ITO)/poly [ethylene dioxythiophene]:poly[styrene sulfonate] (PEDOT:PSS) and aluminum (Al) electrodes. It is, therefore, necessary to understand the influences that lead to the observed improvements, in order to prescribe an optimization procedure based on thermal annealing. In order to shed light on the effect exerted by P3HT in the blend device and, hence, by elimination, on the possible role of PCBM, we have studied ITO/PEDOT/P3HT/Al devices, where the preparation procedure was the same as for the ITO/PEDOT/P3HT:PCBM/Al heterojunction solar cells, except that the active layer comprised P3HT alone, without PCBM.

\section{Materials and Methods}

The 98\% regioregular P3HT (Rieke) and PEDOT:PSS (Bayer) used in this work were taken from the same batches applied for P3HT/PCBM photovoltaic devices with efficiencies above 3\% [14]. The energy level diagram of the ITO/PEDOT/P3HT/Al devices (under nonequilibrium conditions) is shown in Figure 1. The insets give the formula of P3HT and a scheme of the device structure. The highest occupied molecular orbital (HOMO) of P3HT has been estimated to range between 5.1 and $5.2 \mathrm{eV}$. Hereto, we have carried out a space charge limited current (SCLC) analysis of hole only thin film devices [15], cyclic voltammetry [16], and photoelectron spectroscopy [17]. The energy gap estimated from absorption spectroscopy is about $2.1 \mathrm{eV}$; therefore, the lowest unoccupied molecular orbital (LUMO) amounts to about $3.0 \mathrm{eV}$. The work functions of ITO, PEDOT, and $\mathrm{Al}$ are about 4.7, 5.0, and $4.3 \mathrm{eV}$, respectively. The ITO/PEDOT/P3HT/Al heterojunction, therefore, can be considered as a hole only device, since, in both directions of current flow, the hole injection barrier is smaller than the electron injection barrier. The high work function electrode was patterned by etching commercially available ITO-coated glass substrates in acid. The substrates were cleaned in deionized water, acetone, toluene, isopropanol, and a hot ultrasonic bath before etching in oxygen plasma. A PEDOT:PSS water-based solution was spin-coated in a glove box, and the films were dried by heating the substrates on a hot plate. A chloroform-based polymer solution 
(P3HT, $10 \mathrm{mg} / \mathrm{ml}$ ) was then spin-coated in the nitrogen atmosphere of a glove box $\left(\mathrm{O}_{2}-2\right.$ ppm and $\mathrm{H}_{2} \mathrm{O}-$ $0.01 \mathrm{ppm})$. The metal top electrode ( $\mathrm{Al})$ was deposited by thermal evaporation in high vacuum of better than $5 \cdot 10^{-7}$ mbar at a rate of about $0.2 \mathrm{~nm} / \mathrm{s}$. Preparation of solar cells followed the same procedure with the exception that the P3HT solution was replaced by a P3HT:PCBM solution in the weight ratio $1: 3$. All devices were stored in nitrogen atmosphere prior to measurement. $24 \mathrm{ITO/PEDOT/P3HT/Al} \mathrm{devices} \mathrm{prepared}$ in the same batch were annealed at different temperatures, in order to study the effect of annealing and that of the annealing temperature on the electrical behaviour of these devices. Current density versus voltage $(J-V)$ curves for all 24 devices were obtained by using a d.c. current-voltage source/monitor unit (Advantest TR 6143) as a voltage source and current monitor, respectively, with the device placed in a vacuum better than $10^{-5}$ mbar inside a liquid nitrogen-cooled cryostat held at $(290 \pm 0.2) \mathrm{K}$ ambient temperature. Annealing performed at $90{ }^{\circ} \mathrm{C}, 100{ }^{\circ} \mathrm{C}, 130{ }^{\circ} \mathrm{C}, 150{ }^{\circ} \mathrm{C}$ and $5 \mathrm{~min}$ duration was done in batches of six devices. The $J$ - $V$ curves for all 24 devices were measured again at $290 \mathrm{~K}$. In all cases, the voltage sweep extended from negative to positive sign.

\section{Results and Discussion}

\subsection{ITO/PEDOT/P3HT/Al Devices}

The electrical conductivity of P3HT is expected to increase accompanied by increased crystallinity and coplanarity of main chains after heat treatment. The increase of coplanarity of the thiophene rings on a main chain brings the enhancement of the effective conjugation length and the intra-chain carrier mobility. Thus, both the mobility of the charge carriers and the conductivity are expected to increase after heat treatment. However, reduced conductivity has been observed after heat treatment [18].

\section{Low Temperature Annealing}

Figures $2 \mathrm{a}$ and $\mathrm{b}$ show typical dark $J-V$ characteristics of as-cast ITO/PEDOT/P3HT/Al hole only devices in a semi-logarithmic scale, measured at $290 \mathrm{~K}$. High rectification factors of $\sim 5 \cdot 10^{5}$ were observed at $4 \mathrm{~V}$. The values of the current density for devices annealed at $90{ }^{\circ} \mathrm{C}$ and $110{ }^{\circ} \mathrm{C}$ are lower than those for their respective as-cast counterparts. We explain the reduction
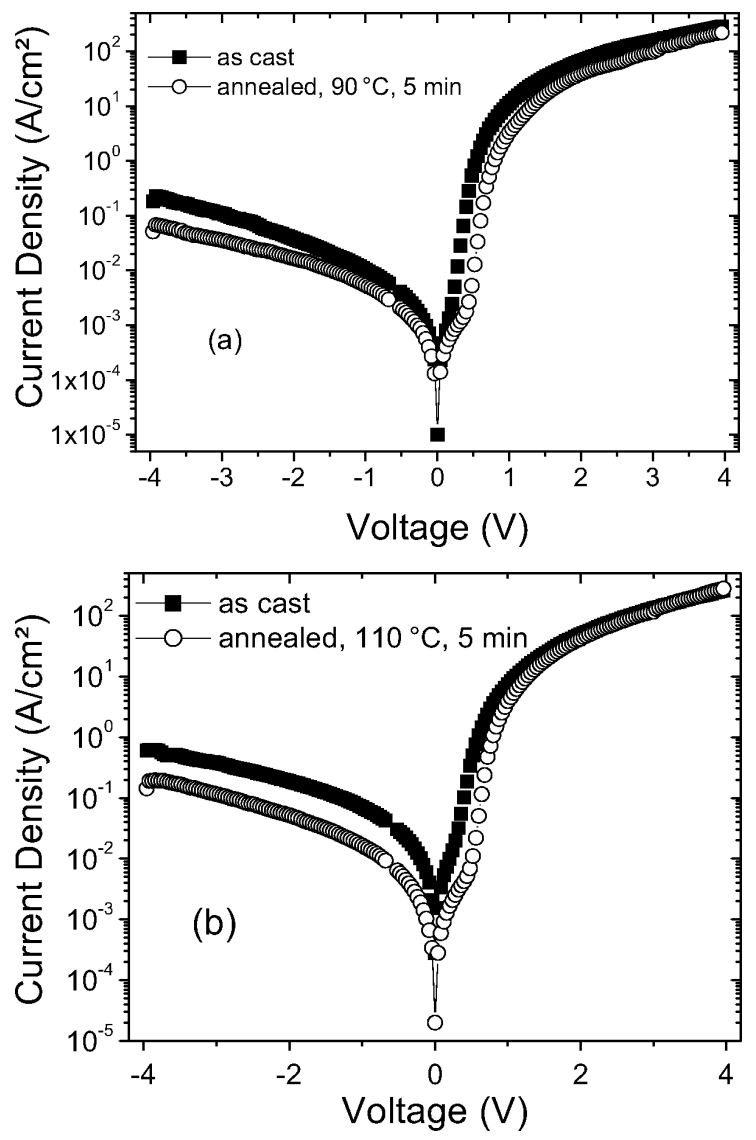

Fig. 2. $J-V$ curves of an ITO/PEDOT/P3HT/Al device in semi-logarithmic scale, measured before and after annealing at (a) $90{ }^{\circ} \mathrm{C}$ for $5 \mathrm{~min}$ and (b) $110{ }^{\circ} \mathrm{C}$ for $5 \mathrm{~min}$. The current density is lower for the annealed device, indicating lower conductivity. The onset of the exponential region is shifted to the right, i. e., to higher voltage.

in current after the annealing step as resulting from dedoping the polymer of impurities such as oxygen, water, and remnant solvent. Dedoping is more severe in the $110{ }^{\circ} \mathrm{C}$ annealed device as compared to the $90{ }^{\circ} \mathrm{C}$ case, hence, the latter exhibits much lower currents.

During production and solution preparation of P3HT, oxygen is absorbed from the environment. Therefore, despite inert ambient-based device fabrication and electrical characterization, significant concentrations of oxygen are expected to be embedded in the films. The oxygen dopant serves to improve the conductivity of P3HT through the production of localized states within the $\pi-\pi^{*}$ gap [13]. Annealing at temperatures below $110{ }^{\circ} \mathrm{C}$ for $5 \mathrm{~min}$ frees the polymer of trapped impurities (e.g., remnant solvent, water, and oxygen), but the annealing temperature and duration 
are below some threshold value of supplied energy, so the polymer chains may remain in their as-cast positions. Oxygen dedoping leads to a decrease in conductivity. We conclude that reduction of free volume and consequent increase of inter-chain interaction are, therefore, negligible under these annealing conditions.

Mattis et al. [13] showed via Rutherford backscattering spectrometry that, indeed, oxygen is one of the substances given off when P3HT thin film devices are heated and that the amount of oxygen given off increases with the annealing temperature. For devices annealed at temperatures higher than $100{ }^{\circ} \mathrm{C}$, they could not detect any remaining traces of oxygen in their samples. Nakazono et al. [18] also suggested that the density of charge carriers induced by the structural defects and by oxygen or moisture may decrease upon heat treatment, leading to some reduction in conductivity. Traps are a particular problem in the fabrication of nchannel semiconductor devices, for example, because oxygen itself is reduced easily enough to act as an electron trap when devices are operated in air [19]. Oxygen captures the electrons and retains them, thereby making available a higher number of holes in the HOMO of P3HT to contribute to the current. The observed decrease in conductivity may, therefore, be interpreted as the reduction in electron trapping centers and, hence, reduction of trap-induced hole density.

\section{High Temperature Annealing}

Devices annealed for $5 \mathrm{~min}$ at $130{ }^{\circ} \mathrm{C}$ and $150{ }^{\circ} \mathrm{C}$ (see Figs. 3a and b, respectively) showed higher, reverse and low voltage forward, current densities as compared to their as-cast counterparts. The increase is considerably stronger for devices annealed at $150{ }^{\circ} \mathrm{C}$. We attribute this finding to film reordering, possibly leading to the crystallization of P3HT. Charge transport through conjugated polymer films requires intimate electrical contact between the polymer segments. The relatively high spin-coating and solvent evaporation speeds tend to dry the polymer chains under tension of the centrifugal and friction forces. Film ordering in as-cast films is poor, since spin-coating tends to leave the polymer chains lying in the plane of the film [20], so that there rarely exists a single polymer chain bridging the electrodes in an electrode/polymer/electrode sandwich device. Thermally annealing a polymer film above its glass transition temperature, $T_{\mathrm{g}}$, allows the freely flowing chains in the polymer melt to untangle into lower energy conforma-
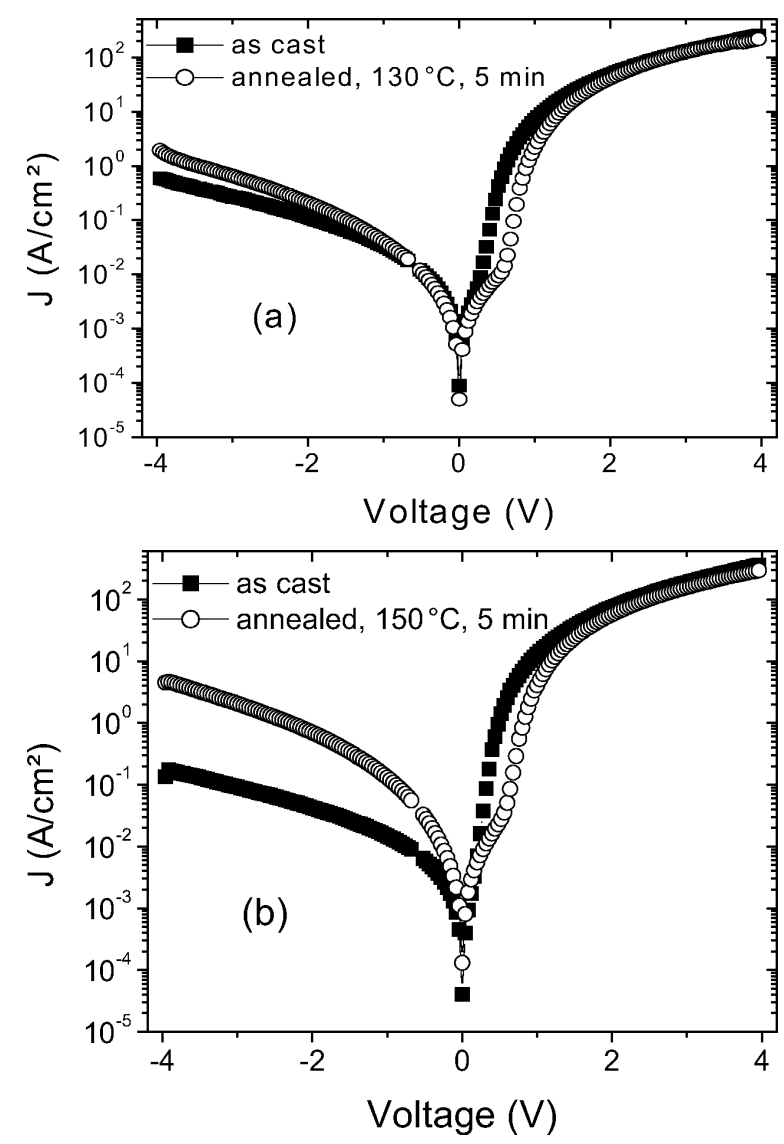

Fig. 3. $J-V$ curves of an ITO/PEDOT/P3HT/Al device in semi-logarithmic scale, measured before and after annealing at (a) $130{ }^{\circ} \mathrm{C}$ for $5 \mathrm{~min}$ and (b) $150{ }^{\circ} \mathrm{C}$ for $5 \mathrm{~min}$. The current density is higher for the annealed device, indicating higher conductivity. The onset of the exponential region is shifted to the right.

tions, and an enhanced crystallization of the polymer may take place [21].

The glass transition temperature of P3HT is around $12{ }^{\circ} \mathrm{C}$ [21], its melting temperature is $220^{\circ} \mathrm{C}$ [22]. The crystal of P3HT is formed by aligned chains which are stacked on top of each other, with the thiophene ring forming a planar alternating 'up-down' conformation and the alkyl side chains pointing perpendicularly to the stacking direction [21]. The presumed straightening of the polymer strands upon annealing would lead to better chain packing and, thus, to an increase in the degree of inter-chain interactions in annealed films relative to as-cast ones; so charge may be conducted along as well as across chains. Thus, along with enhanced crystallization, the hole conductivity of polythiophene increases dramatically [23]. The high mobility in re- 
gioregular P3HT is attributed to the formation of extended polaron states as a result of self-organization in the films [24].

When P3HT saturates with injected charge, the space charge conductivity decreases slightly (see Figs. 2 and 3). Note that the amount of charge, the present material can accommodate when traps are all filled, is now smaller, therefore, the same holds for the amount of charge available for "unlimited" conduction. Annealing at $150^{\circ} \mathrm{C}$ increases the conductivity at low electric fields. After impurity dedoping, the polymer chains also have enough energy to move and "wiggle" into low energy conformations, thereby reordering and possibly increasing crystallinity. The chains then pack closer together, and "dangling bonds" may rejoin, increasing the chain length, and thereby further reducing the trap density. We attribute shallow traps to the impurity states, while deep traps should be due to the unperturbed polymer chain length. Impurities almost disappear upon annealing.

Extremely high temperatures induce conformational changes within $\mathrm{P} 3 \mathrm{HT}$ and may degrade the device performance. The protruding alkyl chains cause some torsioning along the backbone of the molecule. The torsioning of the P3HT chain decreases the conjugation length, and therefore, increases the bandgap, and drastically reduces the conductivity of the film. The physical change is irreversible, permanently harming the electrical characteristics of the device. Further, the differences in the expansion coefficients of the polymer and the inorganic materials constituting the device may lead to buckling which takes place in order to relieve tensile stress, resulting in a wavy metal surface. Annealing at such high temperatures may lengthen the lifetime, but reduce the efficiency [25].

\section{Annealing the Same Device Several Times}

Annealing the same device several times (Fig. 4) shows clearly that annealing is a two-stage process leading first to a decrease in conductivity, followed by an increase of the same. Figure 5 shows the current density versus anneal count for the reverse bias data of Figure 4 . The current first decreases and reaches a minimum. Then it increases, surpassing the as-cast measured current. Both impurity dedoping and film ordering effects may take place simultaneously all the time, but one effect is more effective than the other. The minimum indicates the situation when the effect of impurity dedoping has been overtaken by that of thermally

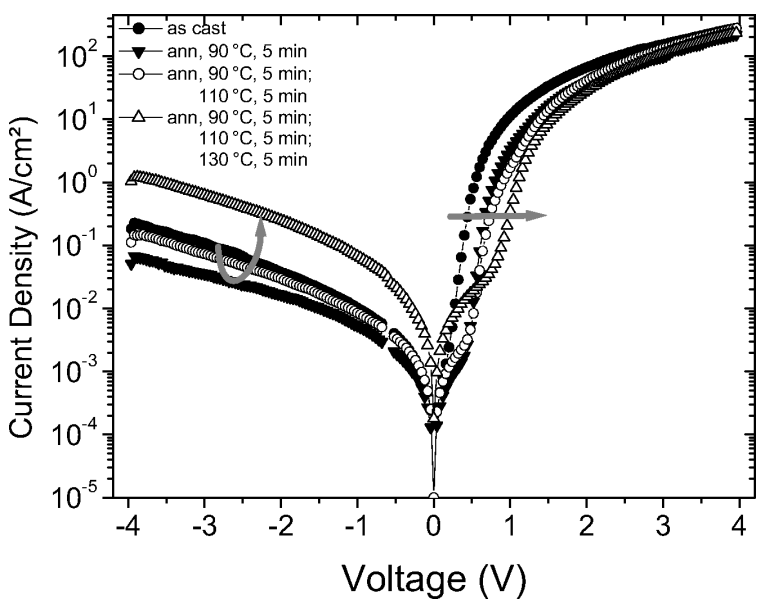

Fig. 4. $J$ - $V$ curves of an ITO/PEDOT/P3HT/Al device in semi-logarithmic scale, measured before and after annealing the same device several times as indicated. Under reverse and low voltage forward bias, the current density first decreases, then increases with each further annealing step, as marked by the curved arrow. The straight arrow indicates a shift of the onset of the exponential region of the curves to high voltages with each further annealing step. The current density is lower at high forward bias voltages.

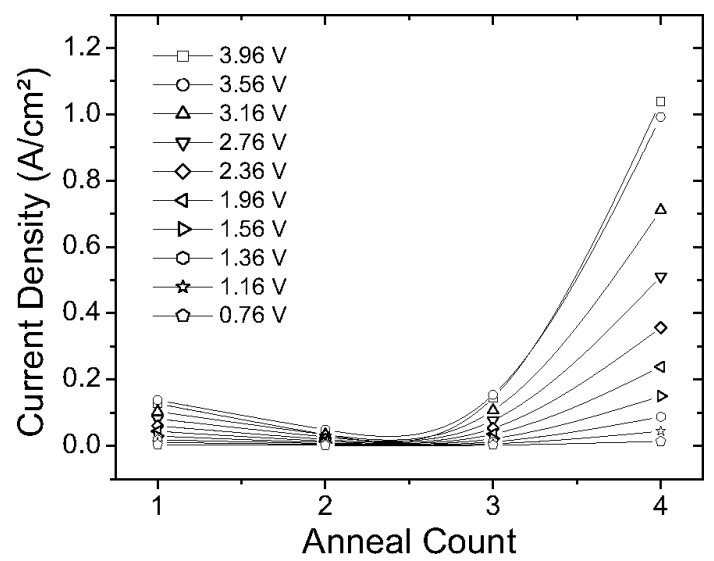

Fig. 5. Current density versus anneal step of an ITO/PEDOT/ $\mathrm{P} 3 \mathrm{HT} / \mathrm{Al}$ device, measured before and after each annealing step (extracted from the data of Fig. 4) at different reverse voltages. The current density first decreases, then increases, indicating that the final result is due to a two-stage process: impurity dedoping, leading to decreasing conductivity; then thermally induced reordering of the P3HT chains, leading to stronger inter-chain interactions and, hence, increasing conductivity.

induced polymer chain reorganization. In the following subsections, we discuss the forward bias curves of the devices before and after annealing.

Figure 6 shows forward bias $J$ - $V$ curves of the devices, which are annealed several times, in a double- 


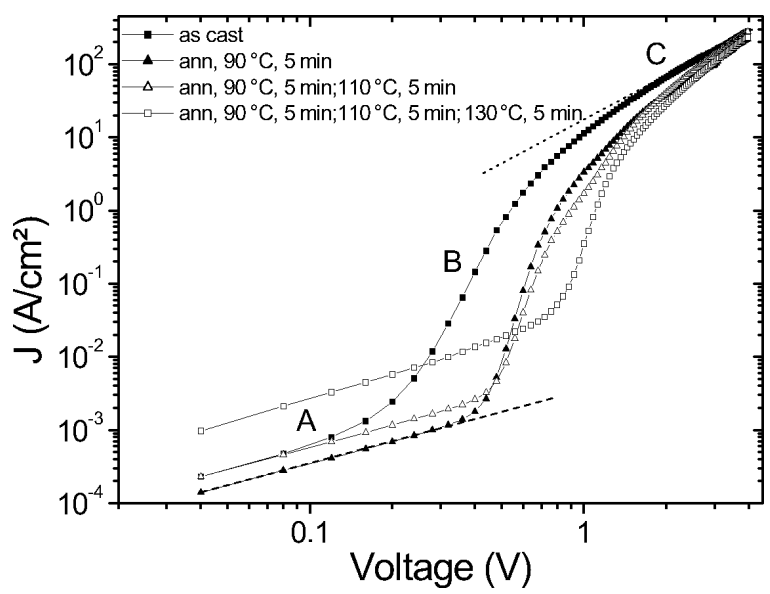

Fig. 6. Dark $J-V$ curves of an ITO/PEDOT/P3HT/Al device in double-logarithmic scale, measured under forward bias, before and after each annealing step. Regions A have a slope equal 1 (dashed line), corresponding to ohmic conduction, regions $\mathrm{B}$ have slopes larger than 2, corresponding to charge injection, and regions $\mathrm{C}$ approach a slope equal 2 (dotted line), corresponding to TFSCLC.

logarithmic representation, corresponding to hole injection through the ITO/PEDOT electrode. The slope of the $J-V$ curve in the above plot provides information on the laws governing the conduction in the device: Slope 1 indicates compliance with Ohm's law, where $J$ is linearly dependent on $V$. Slope 2 indicates trap-free space charge limited current (TFSCLC) conduction. Slope $>2$ indicates the filling of traps distributed exponentially or Gaussianly within the $\pi-\pi^{*}$ gap [26, 27]. A vertical $J-V$ curve in the double-log plot features the interception of traps distributed on a discrete energy level.

At low voltages, the slopes of the $J-V$ curves in the $\log$-log plot of Fig. 6 are about 1 for both the as-cast and annealed device (region A). They increase gradually to larger than 2 (region B), levelling off to 2 at high voltages (region $\mathrm{C}$ ). For the as-cast device, the transition from small slope is gradual, suggesting the filling of traps of continuous distribution in energy. Trap filling starts at lower voltages for the case of the as-cast device, compared to the annealed counterpart, suggesting that the as-cast device has shallow traps. It seems that most of those shallow traps have disappeared in the case of the annealed device, since trap filling now starts at a much higher voltage with each further annealing step. The latter confirms our notion that the density of shallow traps decreases upon annealing and that the observed increase in current after annealing several times derives from another effect, definitely not related to those traps. The transition from ohmic to SCLC behaviour is nearly abrupt, giving rise to an almost vertical section in the double-logarithmic $J-V$ characteristics. Apparently, the traps are more defined in energy. Vertical $J-V$ curve means that traps are situated in a discrete energy level within the $\pi-\pi^{*}$ gap.

\section{Barrier Modification}

Annealing modifies the ITO/PEDOT/P3HT hole injection barrier. Such an effect may be understood through employment of the Fowler-Nordheim (FN) theory, which predicts that if charge injection from the electrodes is governed by quantum mechanical tunneling through a triangular interface barrier, then a plot of $\ln \left(J / F^{2}\right)$ versus $1 / F$ yields a straight line, the slope of which is directly proportional to $\phi^{3 / 2}$, where $\phi$ denotes the potential barrier at that interface [28]. $F=V / d$ means the applied electric field, $V$ the applied voltage, and $d$ the thickness of the device. Additionally, it has been demonstrated that the total current flowing in a device may be modelled by the sum of the contributions due to charges that are injected through tunnelling (FN mechanism) and thermionically over the barrier (Richardson-Schottky mechanism), where, for a constant temperature, the thermionic contribution may be considered constant. Such consideration yields a minimum in the FN plots delimiting the transition from thermionic- to tunneling-dominated injection as the electric field increases [29]. The thermionic effect becomes dominant at high temperatures and low fields, while the tunneling effect provails at low temperatures and high fields.

Figure 7 shows FN curves obtained from the data of Fig. $3 \mathrm{~b}$ for the case of about $70 \mathrm{~nm}$ thick devices. They consist of straight line portions at high fields (tunneling) and curved portions at low fields (thermionic emission). At relatively high electric fields, the curves deviate from straight lines, because there is so much injected charge which overwhelms the transport capabilities of the polymer and, hence, builds up space charge which resists further charge carrier injection. The slopes of the straight parts are clearly larger for the annealed device compared to the as-cast counterpart. The slopes of the FN curves each time become larger upon further annealing, therefore, the ITO/PEDOT/P3HT hole injection barrier increases. The latter holds for the hole annealing temperature range examined (from 90 to $150{ }^{\circ} \mathrm{C}$ ). On the other hand, it is clear that the thermionic contribution is larger for the as-cast device 


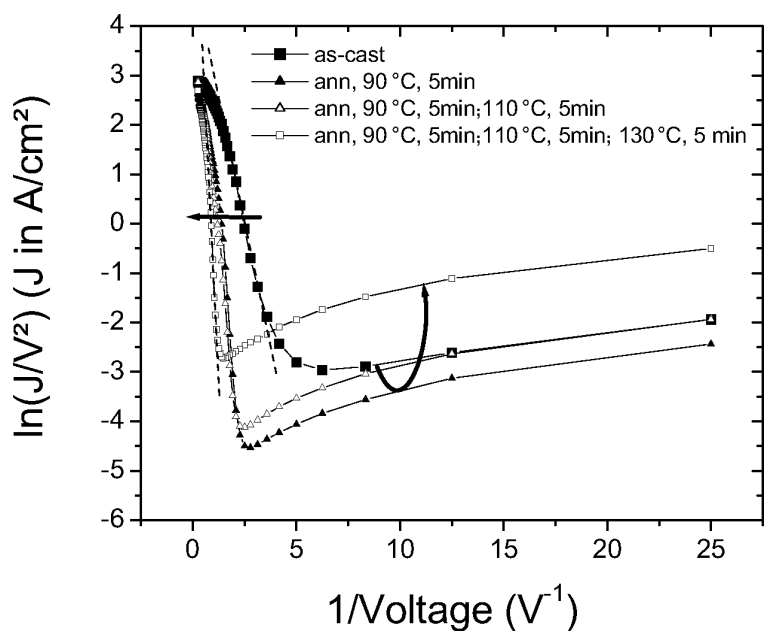

Fig. 7. Fowler-Nordheim plots for hole injection into an ITO/PEDOT/P3HT/Al device through the ITO/PEDOT electrode, measured before and after each annealing step. The slope of the straight line region (dashed lines) increases after annealing, indicating a corresponding increase in the hole injection barrier. The curved parts of the FN plots indicate the current contribution of thermionically injected holes. This current, related to thermally generated charge carriers, decreases after the first annnealing step, then increases subsequently upon further annealing.

than for the case where the device is annealed at $90{ }^{\circ} \mathrm{C}$, supporting our suggestion that the number of thermally generated charge carriers available for conduction is higher in that device, because the impurities in the bulk of the as-cast P3HT film immobilize electrons. It also confirms the idea that such traps are shallow and can be filled thermally even at $290 \mathrm{~K}$. The number of free holes is, therefore, high for as-cast devices.

At high forward voltages above $2 \mathrm{~V}$, as-cast and annealed $J-V$ curves coincide. One would expect, therefore, to get the same space charge limited characteristics. Although the intrinsically generated hole density increases upon annealing the devices at high temperatures, it is equally negligible at high voltages, and space charge limits the current in much the same way as in as-cast devices. However, for the most interesting region for optoelectronic devices like light emitting diodes and solar cells, most of the shallow traps have disapperared upon annealing.

It is important to note that both the impurity dedoping process and thermally induced chain re-ordering may take place at the same time in P3HT-based devices at the temperatures applied. Keep in mind that the glass transition temperature of $\mathrm{P} 3 \mathrm{HT}$ is about $12{ }^{\circ} \mathrm{C}$ and limited film ordering should start to take place around such a temperature. However, it is not the dominant effect at low temperatures and for the duration of annealing employed. At high temperatures, evaporation of remnant solvent, water vapour, and oxygen dedoping should rapidly take place, and the polymer chains receive more thermal energy, allowing them some significant reordering.

\subsection{ITO/PEDOT/P3HT:PCBM/Al Solar Cells}

ITO/PEDOT/P3HT:PCBM/Al heterojunction solar cells were prepared in exactly the same way as the P3HT devices discussed above. The active layer consisted of $1: 3$ (mass ratio) P3HT:PCBM in a $10 \mathrm{mg} / \mathrm{ml}$ chloroform solution. We have measured external quantum efficiencies (EQE) of the cells at $290 \mathrm{~K}$ before determining their $J-V$ characteristics. They were then annealed at $120^{\circ} \mathrm{C}$ for $2 \mathrm{~min}$ in a glove box with nitrogen atmosphere. Afterwards, EQE and $J-V$ were determined again at $290 \mathrm{~K}$. In Fig. 8, we present the $\mathrm{EQE}$ spectra of an as-cast and subsequently annealed ITO/PEDOT/P3HT:PCBM/Al solar cell. The area under the EQE curves gives the short circuit current density. EQE increase dramatically upon annealing these devices at $120{ }^{\circ} \mathrm{C}$ for $2 \mathrm{~min}$, resulting in a short circuit current density increase from 1.84 to $5.17 \mathrm{~mA} / \mathrm{cm}^{2}$ at $100 \mathrm{~mW} / \mathrm{cm}^{2}$ white light illumination.

Figure 9 shows the dark $J$ - $V$ characteristics before and after annealing the solar cells. The current decreases by more than one order of magnitude under

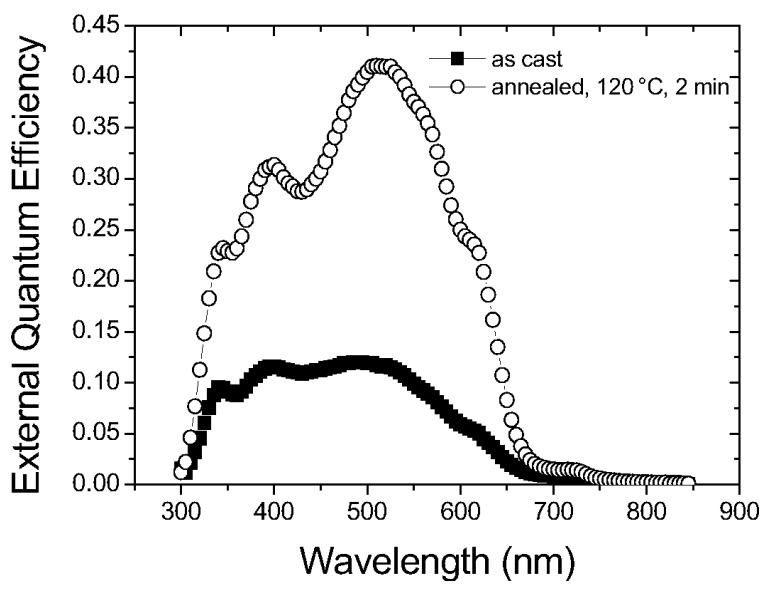

Fig. 8. EQE spectra of $1: 3$ (mass ratio) ITO/PEDOT/ P3HT:PCBM/Al solar cell, measured before and after annealing for $2 \mathrm{~min}$ at $120{ }^{\circ} \mathrm{C}$. The area under the curve gives the short circuit current density, which increases from 1.84 to $5.17 \mathrm{~mA} / \mathrm{cm}^{2}$ for an AM1.5 solar spectrum. 


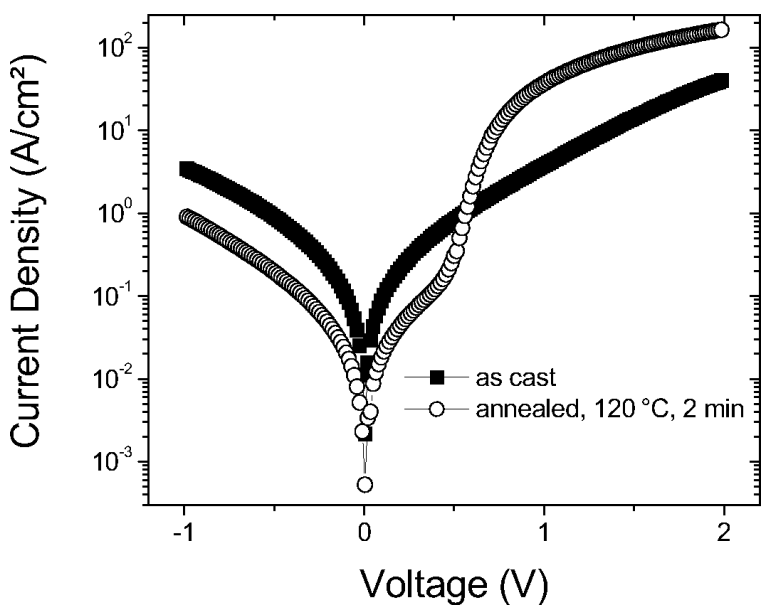

Fig. 9. Dark $J-V$ curves of an ITO/PEDOT/P3HT:PCBM/Al solar cell in semi-logarithmic scale, measured before and after annealing for $2 \mathrm{~min}$ at $120{ }^{\circ} \mathrm{C}$. The current density is lower for the annealed cell under reverse bias and also under low forward bias voltages before the onset of the exponential range.

reverse bias, and also under forward bias before the onset of the exponential region. Such a decrease is in conformity with the effects observed for P3HT devices annealed at 90 and $110{ }^{\circ} \mathrm{C}$ (see Fig. 2). However, at high forward voltages, the annealed device exhibits a much higher current, also by close to one order of magnitude. The decrease in reverse bias current and the increase in forward bias current result in strongly enhanced rectification factors, which expand from 1.1 to 401.1 (i. e., 400 times) at $1 \mathrm{~V}$. The largest increase in forward current takes place just after the exponential region, suggesting that at some high voltage the two curves may actually merge as in the case of the P3HT devices (see Figs. 2, 3, and 4). Polymer ordering temperatures may not have been reached by annealing the devices at $120{ }^{\circ} \mathrm{C}$ for $2 \mathrm{~min}$.

FN plots of the dark $J-V$ characteristics indicate straight lines at high voltages, assuming tunnelling of holes into the HOMO of P3HT under forward bias. These plots illustrate a larger slope for the annealed device compared to the as-cast counterpart, similar to the behaviour observed for P3HT devices. The hole injection barrier (ITO/PEDOT/P3HT), therefore, increases after annealing the solar cell. These annealing effects may, thus, be triggered by barrier modification and impurity dedoping. The pronounced increase in external quantum efficiency and, hence, short circuit current density cannot be explained by a mobility increase in the blend. If it increases at
Table 1. Output characteristics of an ITO/PEDOT/ P3HT:PCBM/Al solar cell at $100 \mathrm{~mW} / \mathrm{cm}^{2}$ white light illumination, measured at $290 \mathrm{~K}$, before and after annealing the cell at $120{ }^{\circ} \mathrm{C}$ for $2 \mathrm{~min}$. The percentage increase of each parameter is also indicated.

\begin{tabular}{lcclc}
\hline & $J_{\mathrm{sc}}\left(\mathrm{mA} / \mathrm{cm}^{2}\right)$ & $V_{\mathrm{oc}}(\mathrm{V})$ & $\mathrm{FF}(\%)$ & eff $(\%)$ \\
\hline As-cast & 1.835 & 0.485 & 28.684 & 0.255 \\
Annealed & 5.170 & 0.605 & 53.338 & 1.669 \\
\% increase & 181.9 & 24.7 & 86 & 554.5 \\
\hline
\end{tabular}

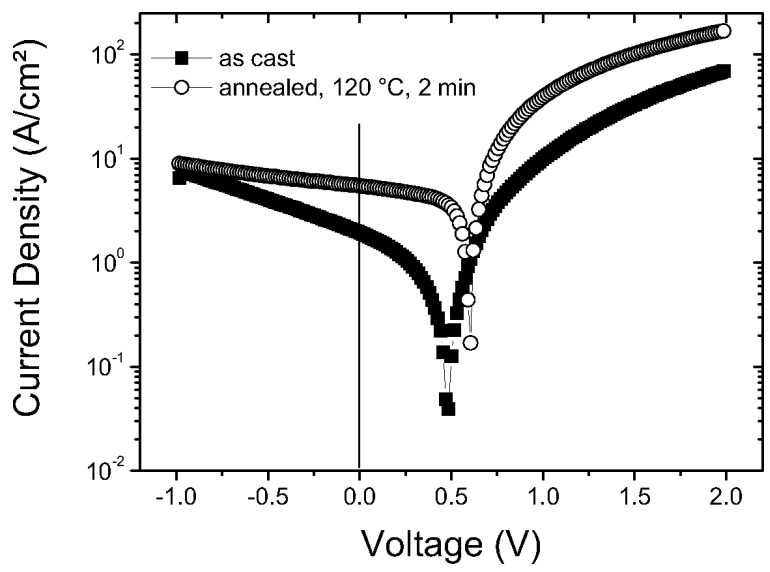

Fig. 10. $J-V$ curves of an $100 \mathrm{~mW} / \mathrm{cm}^{2}$ white-lightilluminated ITO/PEDOT/P3HT/PCBM/Al solar cell (the one of Figs. 8 and 9) in semi-logarithmic scale, measured before and after annealing at $120^{\circ} \mathrm{C}$ for $2 \mathrm{~min}$. The current density under both reverse and forward bias is higher after annealing. $J_{\mathrm{sc}}$ and $V_{\mathrm{oc}}$ both increase after annealing.

all, then it definitely does not represent the dominant effect.

Figure 10 shows a semi-logarithmic plot of the $J-V$ characteristics obtained from the corresponding $100 \mathrm{~mW} / \mathrm{cm}^{2}$ illuminated cells. Both the open circuit voltage $V_{\mathrm{oc}}$ and the short circuit current density $J_{\mathrm{sc}}$ of the annealed device surpass the relating quantities of the as-cast one. Table 1 gives a comparative summary of the relevant output characteristics of the ITO/PEDOT/P3HT:PCBM/Al solar cell taken before and after annealing.

\section{Implications of Impurity Dedoping}

Increase of the potential barrier implies some difficulty with respect to hole injection into the HOMO of P3HT at low voltages. But because of impurity dedoping, some traps have been emptied, and polaronic states which participated in conduction are not there anymore. There is need for supplying a higher energy to fill up the traps, i. e., to increase the charge density in 
the bulk. Therefore, the detrapped impurities are shallow. Being so, they could be filled thermally even at a temperature of $290 \mathrm{~K}$. As they disappear, the density of thermally generated charge carriers is reduced. Thus, the reverse and low voltage forward bias currents are smaller for the case of the annealed device. The abrupt increase in forward current observed for annealed solar cells indicates the filling of deep traps distributed in a narrow energy band, which are suggestive of deep traps related to the chain length. Yet, the material can hold only a fixed maximum amount of charge, with or without traps. Although the process of trap filling is slow for the as-cast device when all traps are filled, we expect the same TFSCLC for both cases. We can, therefore, conclude that the charge carrier density or the capacity for injection at low voltages has been affected, but not the mobility.

It is not expected that electrons trapped by $\mathrm{O}_{2}$ can participate at the current, instead, they are immobilized, giving the polymer some chance to remain with a high number of holes for conduction. These holes cannot recombine with the trapped electrons. Once trapping centers are reduced, the number of recombination events in the polymer increases, thereby decreasing the hole density. PCBM may not trap such low energy electrons. Hence, it is unlikely that they could be transferred to PCBM. It captures only excited electrons that have reached the LUMO of P3HT. Therefore, dedoping the polymer of oxygen, water, and remnant solvent does not increase the mobility of charge carriers. It merely reduces the density of holes participating at the current, which, thus, decreases.

Impurity dedoping can have positive implications for conductivity when the ITO/PEDOT/ $\mathrm{P} 3 \mathrm{HT}$ :PCBM/Al solar cell is illuminated. Ultrafast photo-induced electron transfer from P3HT to PCBM occurs with an efficiency close to $100 \%$ [30]. Both holes and the electrons participate at the current. The overall effect is that the number of electron transfers to PCBM increases, since fewer electrons are captured and immobilized as in the case of abundant oxygen presence. We expect that the current should be higher in the illuminated annealed device. The failure to reach $100 \%$ electron transfer efficiency may be related to the remaining impurity materials that compete with PCBM, but immobilise the electrons captured. The number of electrons conducted through the PCBM LUMO has increased. It may balance the number of those being conducted through the P3HT LUMO. The number of scattering centers decreases, and the

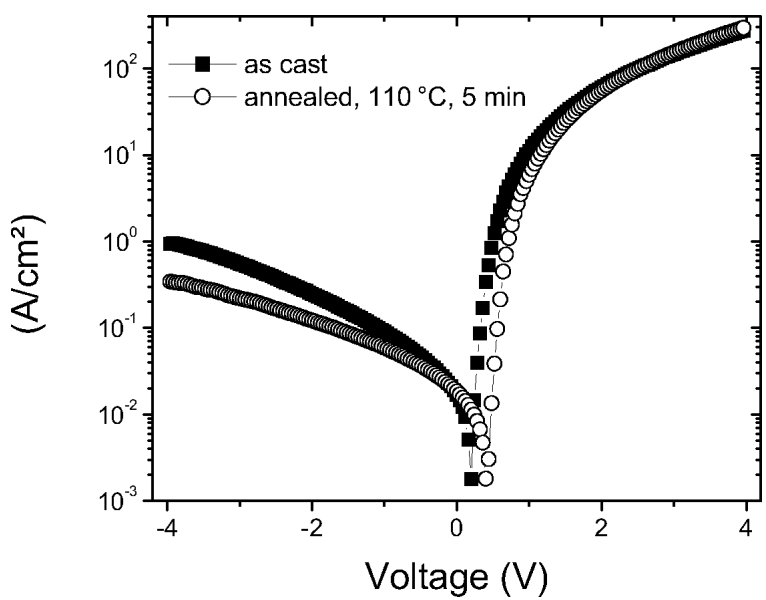

Fig. 11. $J-V$ curves of an $100 \mathrm{~mW} / \mathrm{cm}^{2}$ white-lightilluminated ITO/PEDOT/P3HT/Al device (the one of Fig. 2b) in semi-logarithmic scale, measured before and after annealing at $110^{\circ} \mathrm{C}$ for $5 \mathrm{~min}$. The current density under both reverse and forward bias is lower after annealing. $J_{\mathrm{sc}}$ and $V_{\mathrm{oc}}$ both increase after annealing.

overall conductivity increases. We suggest that the major contributor to the latter is the increase in charge carrier density participating at the current, rather than that in mobility. Keep in mind that, for the dark $J-V$ characteristic, the current decreases anyway, even if the number of scattering centers has also become smaller. Figure 11 shows $J$ - $V$ curves obtained from the illuminated ITO/PEDOT/P3HT/Al device of Fig. 1b before and after annealing at $110{ }^{\circ} \mathrm{C}$ for $5 \mathrm{~min}$. Note that the illuminated current is lower for the case of the annealed device. The reason is that there does not exist any additional charge transfer and, although the number of holes increased due to photo-excitation, it has not reached the same level as if impurities simply immobilized the electrons.

Further, thermally induced interdiffusion may increase the overlap of wave functions between P3HT and PCBM, making charge transfer easier. During annealing, a rush of colour is observed after a time span of about $20 \mathrm{~s}$. We attribute the latter to the motion of PCBM molecules as they rush to occupy the sites left by impurities like oxygen and water. Possible crystallization of PCBM may also create easier percolation paths for electrons moving towards the $\mathrm{Al}$ electrode.

High temperature annealing induces stronger interchain interactions which promote bipolaronic recombination of charge carriers. Although strong interchain interactions increase the mobility of holes in the polymer, it has a negative effect on quantum ef- 
ficiency. Padinger et al. [5] observed that annealing P3HT:PCBM solar cells at $75{ }^{\circ} \mathrm{C}$ increases the quantum efficiency to some maximum within 5 to $6 \mathrm{~min}$, but any longer annealing period at the same temperature decreases the overall efficiency dramatically again. This finding suggests that strong inter-chain interactions may not be as important as dedoping the polymer of oxygen or evaporation of remnant solvent and water. Strong inter-chain interactions also reduce the rectification ratio.

\section{Implications of Barrier Modification on $V_{\mathrm{oc}}$}

We emphasize that the increase of the ITO/PEDOT/ $\mathrm{P} 3 \mathrm{HT}$ hole injection barrier implies an increase in the internal electric field which favours the motion of holes from the P3HT HOMO to PEDOT and, ultimately, to ITO. Accordingly, we have a more effective blocking of electrons and an efficient collection of holes by ITO through PEDOT at zero bias. $V_{\text {oc }}$ increases after thermal annealing (Figs. 10 and 11). Charge injection becomes more difficult, but collection improves.

\section{Conclusions}

Thermal annealing of P3HT films occurs via two stages, the first of which is impurity dedoping leading to some reduction of conductivity, the second of which involves thermal motion of polymer chains resulting

[1] J. Shinar, Organic Light Emitting Devices: A Survey, American Institute of Physics, New York 2002.

[2] C. J. Brabec, V. Dyakonov, J. Parisi, and N. S. Sariciftci, Organic Photovoltaics: Concepts and Realization, Springer, Berlin 2003.

[3] T. Markvart and L. Castaner, Practical Handbook of Photovoltaics: Fundamentals and Applications, Elsevier, Amsterdam 2003.

[4] C. D. Dimitrakopoulos and J. D. Mascaro, IBM J. Res. Develop. 45, 11 (2001).

[5] F. Padinger, R. S. Rittberger, and N. S. Sariciftci, Adv. Funct. Mater. 13, 1 (2003).

[6] H. Sirringhaus, N. Tessler, and R. Friend, Science 280, 1741 (1998).

[7] I. D. Parker, J. Appl. Phys. 75, 1656 (1994).

[8] D. Chirvase, J. Parisi, J.C. Hummelen, and V. Dyakonov, Nanotechnology 15, 1317 (2004).

[9] M. T. Rispens, A. Meetsma, R. Rittberger, C. J. Brabec, N. S. Sariciftci, and J. C. Hummelen, Chem. Commun. 17, 2116 (2003). in reordering and, possibly, crystallization, eventually ending up with an increase in conductivity. We have demonstrated that the critical parameters of annealing temperature and duration, at which strong improvement in external quantum efficiency of P3HT:PCBM bulk heterojunction solar cells is achieved, are generally quantified within the range where the polymer is dedoped of oxygen. We, therefore, propose that increase in mobility due to reordering of $\mathrm{P} 3 \mathrm{HT}$ is not the dominant effect in the present case. When oxygen leaves the film, the number of immobilized electrons decreases and, hence, the number of electrons participating at the generation of current increases. The pronounced increase in quantum efficiency of the ITO/PEDOT/P3HT:PCBM/Al heterostructure observed after some annealing step has, thus, been interpreted in terms of a defined physical ordering mechanism.

\section{Acknowledgements}

The authors acknowledge the Deutsche Gesellschaft für Technische Zusamenarbeit (GTZ), the Deutscher Akademischer Austauschdienst (DAAD), and the German Ministry for Education and Research (BMBF) (projects 01SF0026, 01SF0119) for funding their work. They also thank Verena Mertens, Dana Chirvase, Susanne Böger, Michael Pientka, and Ingo Riedel (all from University of Oldenburg) for valuable contributions and fruitful discussions.

[10] Y. Zhao, G. X. Yuan, P. Roche, and M. Leclerc, Polymer 36, 2211 (1995).

[11] T. Ahn, H. L. Lee, and S.-H. Han, Appl. Phys. Lett. 80, 392 (2002).

[12] J. Liu, T. F. Guo, and Y. Yang, J. Appl. Phys. 91, 1595 (2002).

[13] B. A. Mattis, P.C. Chang, and V. Subramanian, Mat. Res. Soc. Symp. 771, L10.35.1 (2003).

[14] I. Riedel and V. Dyakonov, Phys. Status Solidi A 201, 1332 (2004).

[15] D. Chirvase, Z. Chiguvare, M. Knipper, J. Parisi, V. Dyakonov, and J. C. Hummelen, Synth. Met. 138, 299 (2003).

[16] R. Valaski, L. M. Moreira, L. Micaroni, and I. A. Hümmelgen, J. Appl. Phys. 92, 2035 (2002).

[17] M. Onoda, K. Tada, A. A. Zakhidov, and K. Yoshino, Thin Solid Films 331, 76 (1998).

[18] M. Nakazono, T. Kawai, and K. Yoshino, Chem. Mater. 6, 864 (1994).

[19] G. Horowitz, Adv. Mater. 10, 365 (1998). 
[20] D. McBranch, I. H. Campbell, D. L. Smith, and J.P. Ferraris, Appl. Phys. Lett. 66, 1175 (1995).

[21] Y. Zhao, G. X. Yuan, P. Roche, and M. Leclere, Polymer 36, 2211 (1995).

[22] C. Yang, F.P. Orfino, and S. Holdcroft, Macromolecules 29, 6510 (1996).

[23] J. J. Dittmer, E. A. Marseglia, and R. H. Friend, Adv. Mater. 12, 1270 (2000).

[24] D. Fichou, J. Mater. Chem. 10, 571 (2000).

[25] J. Kim, J. Lee, C. W. Han, N. Y. Lee, and I.-J. Chung, Appl. Phys. Lett. 82, 4238 (2003).
[26] M. A. Lampert and P. Mark, Current Injection into Solids, Academic Press, New York 1970.

[27] K. C. Kao and W. Hwang, Electrical Transport in Solids, Vol. 14, Pergamon Press, Oxford 1981.

[28] R. H. Fowler and L. Nordheim, Proc. R. Soc. London A 119, 173 (1928).

[29] Z. Chiguvare, J. Parisi, and V. Dyakonov, J. Appl. Phys. 94, 2440 (2003).

[30] P. Schilinsky, C. Waldauf, and C. J. Brabec, Appl. Phys. Lett. 81, 3885 (2002). 